\title{
New Analytical Method with Curvature Based Kinematic Deflection Curve Theory Gökhan Tansel Tayyar ${ }^{1^{*}}$ \\ ${ }^{1}$ Faculty of Naval Architecture and Ocean Engineering, Istanbul Technical University, 34469 Maslak, Istanbul, Turkey
}

(Manuscript Received June 11 2012; Revised July 14, 2012; Accepted August 7, 2012)

\begin{abstract}
This paper reports a new analytical method to calculate the planar displacement of structures. The cross-sections were assumed to remain in plane and the deflection curve was evaluated using the curvature values geometrically, despite being solved with differential equations. The deflection curve was parameterized with the arc-length of the curvature values, and was taken as an assembly of chains of circular arcs. Fast and accurate solutions of complex deflections can be obtained easily. This paper includes a comparison of the nonlinear displacements of an elastic tapered cantilever beam with a uniform moment distribution among the proposed analytical method, numerical method of the theory and large deflection FEM solutions.
\end{abstract}

Keywords: Curvature, Deflection curve, Tapered beam, Displacement

\section{Introduction}

Deflection calculation methods of one dimensional (1-D) structures mainly use a deflection curve model by differential equations under some geometrical assumptions. On the other hand, there is an alternative model that the deflection curve is generated geometrically where the deflection curve consists of circular arcs of osculating circles [1].

Geometrical-based theory does not deal with the loads or material model that generates a curvature, which can be calculated from equilibrium equations. The kinematic relationship between curvature and deflection does not change even if material or geometrical nonlinearity occurs. Curvature physically indicates the arc of a circle. Therefore, it models the deflection curve with an arc length parameterization whose direction and radius are determined from the curvature values. In theory, there is only one unit tangent vector [2] and only one unit normal vector for a common point of two adjacent arcs [1].

The main motivation of this approach was to obtain more accurate and faster solutions from deflection

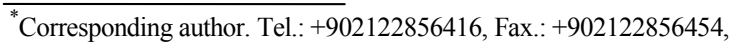

E-mail address: tayyargo@itu.edu.tr

Copyright $\odot$ KSOE 2012.
}

based solutions. The solutions will more accurate because there is no geometrical assumption like in nonlinear deflection theory or large deflection theory, and will also be faster because only pre-prepared curvature diagrams or functions can be used in simple equations without the need to solve differential equations. In addition to the numerical approximation from theory, where the structure was divided into sections [1], a new analytical method is proposed.

A search of the "ISI-Web of Science" did not reveal any study on how to obtain analytically the deflection curve with the geometrical use of inconstant curvature values. The range of this study is limited to the planar motion of structures for simplicity. The Poisson's effect, shear effect and torsion were not considered in the proposed theory. 1-D structures, such as rods, beam columns, and stiffened plates, can be included in this category.

Section 2 presents the kinematic relation between the deflection curve and curvature. The method is illustrated in section 3. The application of the method and a comparison with other solutions are reported in section 4. The last section is devoted to a conclusion.

\section{Deflection Curve}


The deflection curve or centroidal axis of a deformed/undeformed structure was defined by the general regular skew curve: $\boldsymbol{\alpha}$. The structure was generated by cross section when the centroid of the cross section, $C$, moves along $\boldsymbol{\alpha}$ with the plane of the cross section, $A$, being normal to deflection curve.

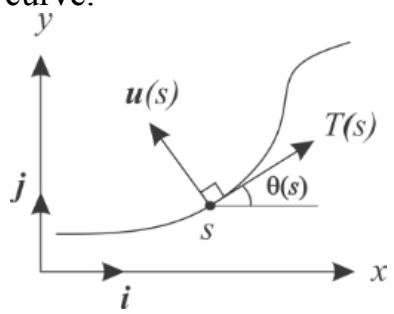

Fig. 1. Unit tangent vector of a deflection curve at a point.

A point on a regular curve has two important unit vectors: unit tangent and unit normal vectors. The direction of the tangent of a point on the deflection curve is determined with the unit tangent vector, $\boldsymbol{T}$, which can be written in the form of $\theta$, the slope angle between the $x$-axis, where $\boldsymbol{i}$ and $\boldsymbol{j}$ are the unit vectors of the $x$ - and $y$-axis, respectively (Eq. (1)). Rectangular Cartesian coordinates and curvilinear coordinates were used. $s$ is the arc length measured along a cross section from one end of the structure to $C$. The normal of the unit tangent vector is called the unit normal vector, $\boldsymbol{u}$, by rotating the tangent vector counterclockwise $90^{\circ}$ (Eq. (2)). These vectors are both differentiable.

$$
\begin{aligned}
& \boldsymbol{T}(s)=\cos (\theta(s)) \boldsymbol{i}+\sin (\theta(s)) \boldsymbol{j} \\
& \boldsymbol{u}(s)=-\sin (\theta(s)) \boldsymbol{i}+\cos (\theta(s)) \boldsymbol{j}
\end{aligned}
$$

Let $I$ be an interval and a continuous function $\alpha$ : $I$ $\rightarrow \mathbb{R}^{3}$ is the arc length parameterized curve defined by a position vector $\boldsymbol{\alpha}(s)=x(s) \boldsymbol{I}+y(s) \boldsymbol{j}+0 \boldsymbol{k}$. Each component is differentiable and $s \in I$ [3]. A curve is regular if the vector $\boldsymbol{\alpha}^{\prime}(s) \neq 0$, i.e. $\left\|\boldsymbol{\alpha}^{\prime}(s)\right\| \neq 0$ [4]. The curvature, $\kappa$, specifying the rotation of this triad as $C$ moves along $\boldsymbol{\alpha}$, was used to describe the configuration of $\boldsymbol{\alpha}$. The curvature can be written in the form of Eq. (3) to express the rate of change of the unit tangent with respect to the arc length $[4,5]$. The curvature indicates a physical shape, an arc of an osculating circle. The radius of the osculating circle of $\boldsymbol{\alpha}(s)$ can be determined using Eq.(4) [5].

$\kappa(s)=(\mathrm{d} \theta(s)) / \mathrm{d} s$ $r(s)=|1 / \kappa(s)|$

If the curvature value has a positive sign, the slope angle will be cumulative and the center of curvature will be aligned by the unit normal vector. In the case of negative curvature values, the deflection shape will be concave and the center of curvature is aligned by the reverse of the unit normal vector. Curvature reports two things. The first is the radius of curvature and the second is the direction of the curvature center. A radius vector, $\boldsymbol{R}$, can be defined from a point on the deflection curve to the center of curvature. $\boldsymbol{R}$ of a point on the deflection curve has a magnitude that can be determined from Eq. (4), and has a direction that can be specified from the sign of curvature and unit normal vector.

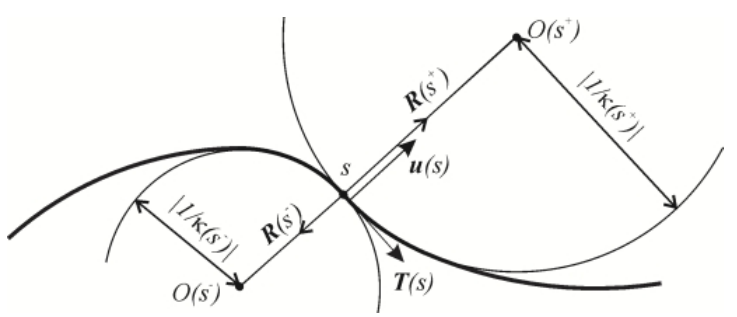

Fig. 2. Osculating circles of a point.

The curvature values are dependent on the internal loads and material properties. Therefore, the curvature function on a deflection curve could be discontinuous. The left and right hand side values of the $\boldsymbol{R}(s)$ vector of a point on a deflection curve can be variable and are expressed as follows (Fig. (3))

$$
\begin{aligned}
& \boldsymbol{R}\left(s^{-}\right)=\boldsymbol{u}(s) / \kappa\left(s^{-}\right) \\
& \boldsymbol{R}\left(s^{+}\right)=\boldsymbol{u}(s) / \kappa\left(s^{+}\right)
\end{aligned}
$$

\section{Displacement Determinations}

Suppose that the internal loads between a point on $s_{1}$ and a point on $s_{2}$ are known and constant. Therefore, the curvature value between a point on $s_{1}$ and a point on $s_{2}$ is assumed to be constant and taken as the curvature of the right side of point $s_{1}: \kappa\left(s_{1}{ }^{+}\right)$.

If the curvature is constant between two points of a deflection curve, all points will have the same radius of curvature and the same center of curvature; sharing the same osculating circle between these points. The deflection curve between a point on $s_{1}$ and a point on $s_{2}$ forms an arc. The radius of the arc is equal to $\left|1 / \kappa\left(s_{1}{ }^{+}\right)\right|$and the center of the arc is on point $O\left(s_{1}{ }^{+}\right)$(Fig. (3)). The vector of the start and 
end point of the arc to the center of $\operatorname{arc}$ is $\boldsymbol{R}\left(s_{1}{ }^{+}\right)$and $\boldsymbol{R}\left(s_{2}{ }^{-}\right)$, respectively.

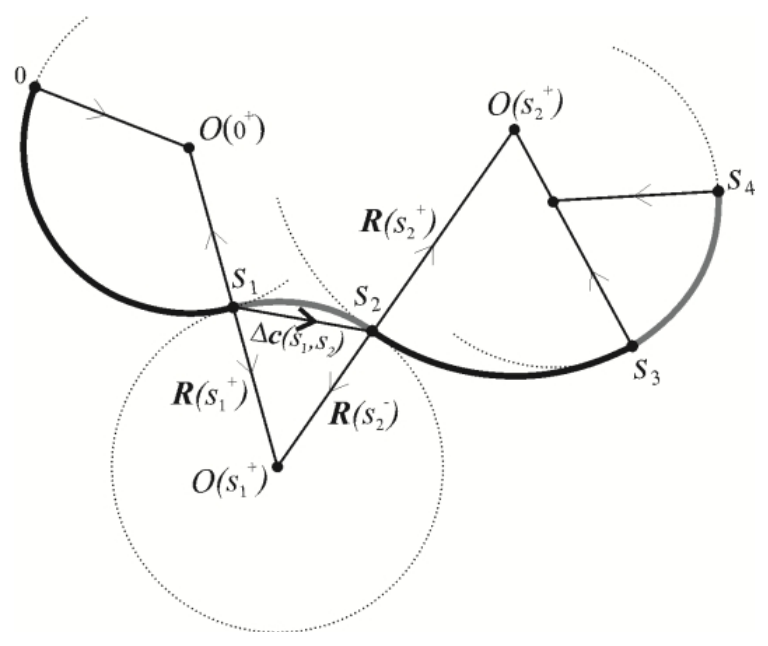

Fig. 3 Radius vectors of the subsequent osculating circles.

$$
\begin{aligned}
& \boldsymbol{R}\left(s_{1}{ }^{+}\right)=\frac{\boldsymbol{u}\left(s_{1}\right)}{\kappa\left(s_{1}{ }^{+}\right)} \\
& \boldsymbol{R}\left(s_{2}^{-}\right)=\frac{\boldsymbol{u}\left(s_{2}\right)}{\kappa\left(s_{2}{ }^{-}\right)}=\frac{\boldsymbol{u}\left(s_{2}\right)}{\kappa\left(s_{1}{ }^{+}\right)}
\end{aligned}
$$

The vector between the start and end points of the arc, $\Delta c$, has a magnitude that equals the chord length of the arc. The displacement vector, $\Delta c$, from point $s_{1}$ to $s_{2}$ can be defined from $\boldsymbol{R}$ vectors as follows (Fig.(3)):

$$
\Delta c\left(s_{1}, s_{2}\right)=\boldsymbol{R}\left(s_{1}{ }^{+}\right)-\boldsymbol{R}\left(s_{2}{ }^{-}\right)
$$

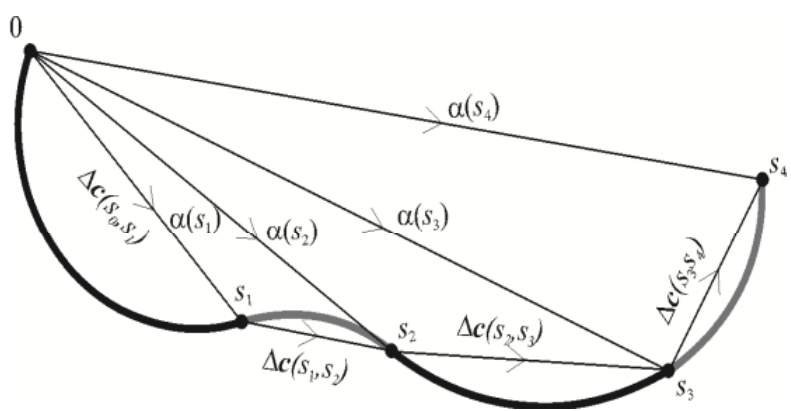

Fig. 4 Displacement and deflection curve vectors of the deflection curve.

The change in the unit normal vector between $s_{1}$ and $s_{2}$ can be defined as follows:

$$
\Delta \boldsymbol{u}\left(s_{1}, s_{2}\right)=\boldsymbol{u}\left(s_{2}\right)-\boldsymbol{u}\left(s_{1}\right)
$$

Eq. (9) can be expressed by substituting Eqs. (7), (8) and (10) into Eq. (9) (Fig. (4)).

$$
\begin{aligned}
\Delta \boldsymbol{c}\left(s_{1}, s_{2}\right) & =\boldsymbol{\alpha}\left(s_{2}\right)-\boldsymbol{\alpha}\left(s_{1}\right) \\
& =-\Delta \mathbf{u}\left(\mathrm{s}_{1}, \mathrm{~s}_{2}\right) / \kappa\left(\mathrm{s}_{1}{ }^{+}\right)
\end{aligned}
$$

The deflection curve can be divided into sections composed of constant curvatures. The displacement vectors of each section can be determined by Eq. (11). The total displacement vector from the origin to point on $s_{\mathrm{n}}$ is equal to the summation of all displacement vectors between the origin point and point $s_{\mathrm{n}}$ as follows:

$$
\begin{aligned}
\boldsymbol{\alpha}\left(s_{n}\right) & =\boldsymbol{\alpha}\left(s_{n}\right)-\boldsymbol{\alpha}(0) \\
& =\Delta \boldsymbol{c}\left(0, s_{1}\right)+\Delta \boldsymbol{c}\left(s_{1}, s_{2}\right)+\cdots+\Delta \boldsymbol{c}\left(s_{n-1}, s_{n}\right)
\end{aligned}
$$

The unit normal vector is differentiable. Therefore, it is possible to take the sections arc lengths as indefinitely small and define the displacement vector in an integral form as follows:

$\boldsymbol{\alpha}(s)=\int_{0}^{\mathrm{s}}-\frac{\mathrm{d} \boldsymbol{u}(\zeta)}{\kappa(\zeta)}$

The unit normal vector in Eq. (2) is dependent on the slope angle: it needs to be defined in terms of the curvature to solve Eq. (12). The slope angle can be expressed in terms of the curvature by integrating Eq. (3) as follows:

$\theta(s)=\theta(0)+\int_{0}^{s} \kappa(\zeta) \mathrm{d} \zeta$

Substituting Eq. (13) into Eq. (2) yields the normal unit vector as follows:

$$
\begin{aligned}
\boldsymbol{u}(s)= & -\sin \left(\theta(0)+\int_{0}^{s} \kappa(\zeta) \mathrm{d} \zeta\right) \boldsymbol{i} \\
& +\cos \left(\theta(0)+\int_{0}^{s} \kappa(\zeta) \mathrm{d} \zeta\right) \mathbf{j}
\end{aligned}
$$

The derivative of the normal unit vector can be obtained from Eq. (14).

$$
\begin{aligned}
d \boldsymbol{u}(s)= & -\cos \left(\theta(0)+\int_{0}^{s} \kappa(\zeta) \mathrm{d} \zeta\right) \kappa(s) d s \boldsymbol{i} \\
& -\sin \left(\theta(0)+\int_{0}^{s} \kappa(\zeta) \mathrm{d} \zeta\right) \kappa(s) d s \boldsymbol{j}
\end{aligned}
$$

Substituting Eq. (15) into Eq. (12) yields the displacement vector from the origin to a point on $s$ as an integration of the curvature function and length.

$\boldsymbol{\alpha}(s)=\boldsymbol{\alpha}(s)-\boldsymbol{\alpha}(0)$ 


$$
\begin{aligned}
= & \int_{0}^{s} \cos \left(\theta(0)+\int_{0}^{\xi} \kappa(\zeta) \mathrm{d} \zeta\right) \mathrm{d} \xi \boldsymbol{i} \\
& +\int_{0}^{s} \sin \left(\theta(0)+\int_{0}^{\xi} \kappa(\zeta) \mathrm{d} \zeta\right) \mathrm{d} \xi \boldsymbol{j}
\end{aligned}
$$

The slope angle of the origin point is essential for starting and obtaining analytical solutions. The points at the clamped ends or extremum points have a zero slope angle. Therefore, these points are practical points to start the procedure. Alternatively, a zero slope angle point can be obtained using a trial and error procedure.

\section{Application}

Three main geometric assumptions are using to calculate the displacements of a deflection curve: linear, nonlinear and large deflections. Consider a sufficiently small segment between points 1 and 2 on the deflection curve: the length between these points is $d s$, the radius of curvature on the segment is equal to $r$ and the chord length between these points is $d c$. The tangent angles at points 1 and 2 are $\theta_{1}$ and $\theta_{2}$, respectively. The central angle of the arc is the difference between the tangent angles $d \theta$ (Fig. (5)).

Consider an elastic tapered cantilever beam under a uniform moment of $M_{\mathrm{z}}$ and a total beam length of $L$ (Fig.(5)). The material of the beam is elastic and the flexural rigidity is $E I$, where $E$ is the elastic modulus, and $I$ is the moment of inertia. The width of the rectangular beam cross-section is $B$, and the height of the beam cross-section is $H_{\max }$ in a clamped edge and $H_{\min }$ in a free end (Fig.(5)). The material of the beam was assumed to be elastic.

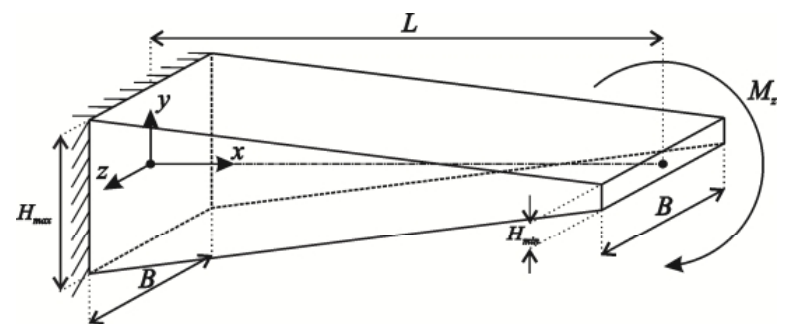

Fig. 5. Elastic tapered cantilever beam

The curvature equation for the elastic rectangular beam cross-section is given as $M_{\mathrm{z}} / E I$ [5]. Therefore, the curvature can be expressed as follows:

$\kappa(s)=-\frac{M_{z}}{E I(s)}=-\frac{12 M_{z}}{E B(H-s \Delta H / L)^{3}}$ where $\Delta H=H_{\max }-H_{\min }$. The displacement equation can be obtained by substituting Eq. (17) into Eq. (16).

$$
\begin{aligned}
\boldsymbol{\alpha}(L) & =\int_{0}^{L} \cos \left(\theta(0)+\int_{0}^{s} \kappa(\zeta) \mathrm{d} \zeta\right) d s \boldsymbol{i} \\
& +\int_{0}^{L} \sin \left(\theta(0)+\int_{0}^{s} \kappa(\zeta) \mathrm{d} \zeta\right) d s \boldsymbol{j}
\end{aligned}
$$

The integration begins from the clamped edge, where $\theta(0)=0$. Therefore, the displacement equation in Eq. (18) can be expressed as follows:

$$
\begin{aligned}
\boldsymbol{\alpha}(L) & =\int_{0}^{L} \cos \left(\int_{0}^{s} \kappa(\zeta) \mathrm{d} \zeta\right) d s \boldsymbol{i} \\
& +\int_{0}^{L} \sin \left(\int_{0}^{s} \kappa(\zeta) \mathrm{d} \zeta\right) d s \boldsymbol{j}
\end{aligned}
$$

The dimensions and main properties are $L=800$ $\mathrm{mm}$, Elasticity modulus $=200000 \mathrm{~N} / \mathrm{mm}^{2}, B=$ $10 \mathrm{~mm}, H_{\max }=12 \mathrm{~mm}$ and $H_{\min }=2 \mathrm{~mm}$, with $M_{\mathrm{z}}$ up to $266667 \mathrm{Nmm}$. Analytical solutions can be obtained easily using Eq. (19), where the curvature is a function described in Eq. (17).

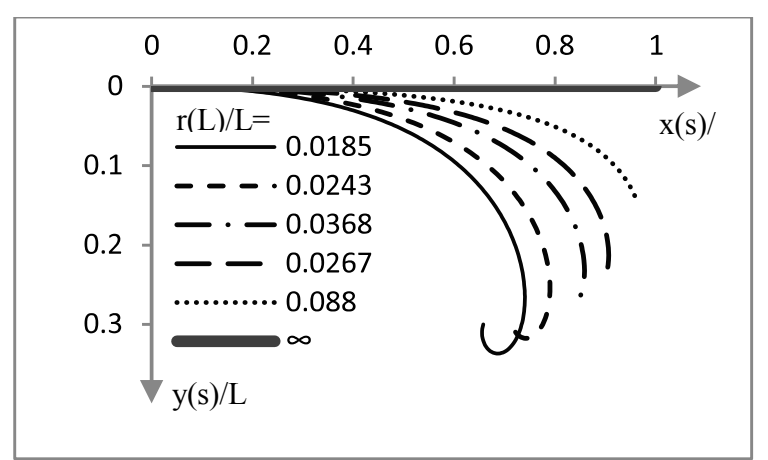

Fig. 6 Deflection shapes of the elastic tapered cantilever beam under uniform moment.

Fig. (6) shows the deflection shapes of the beam with increasing moment. Table 1 lists the results of the converged FEM [1], numerical approximation results of the curvature based displacement theory [1] and proposed method result for the vertical displacement of the free end. The maximum difference between the results of the proposed analytical and the FEM solution was approximately $0.02 \%$. The numerical and analytical methods achieved much closer results; $<0.00001 \%$. 
Table 1. Comparison of the free end vertical displacements.

\begin{tabular}{|c|c|c|c|}
\hline $\mathrm{r}_{\min } / \mathrm{L}$ & $\begin{array}{c}\text { FEM } \\
-y_{\text {free }} / \mathrm{L} \\
\end{array}$ & $\begin{array}{c}\text { Numerical } \\
-\mathrm{y}_{\text {free }} / \mathrm{L}\end{array}$ & $\begin{array}{c}\text { Analytical } \\
-y_{\text {free }} / \mathrm{L} \\
\end{array}$ \\
\hline 0.0062 & 0.33120 & 0.33117 & 0.33117 \\
\hline 0.0125 & 0.31851 & 0.31847 & 0.31847 \\
\hline 0.0187 & 0.30058 & 0.30058 & 0.30058 \\
\hline 0.0250 & 0.30845 & 0.30842 & 0.30842 \\
\hline 0.0375 & 0.27903 & 0.27898 & 0.27898 \\
\hline 0.0625 & 0.20004 & 0.20000 & 0.20000 \\
\hline 0.1000 & 0.13326 & 0.13324 & 0.13324 \\
\hline 0.1875 & 0.07321 & 0.07320 & 0.07320 \\
\hline
\end{tabular}

\section{Conclusions}

Theory provides an opportunity to form the most complex deflection shapes analytically with few inputs, and without the need to solve differential equations. In addition, this method makes the solution fast because the equations are simple. The common assumptions in deflection modeling are not used. Therefore, the solutions are more realistic as observed from results of the application.

Equilibrium can be satisfied only if the axial/inplane stresses are defined precisely. Hence, correct deflection modeling is essential. In addition, second-order theory must be considered with accurate deflection modeling for reliable results in post buckling or in post collapse. Therefore, this theory will be an advance tool for post buckling and post collapse analysis.
This paper focused only on 1-D structures. Nevertheless, the theory can be extended to two dimensional (2-D) structures including compatibility effects. Future studies will focus on developing a procedure for buckling and post buckling calculations of 1-D structures, including an effective width determination as a function of deflection, and applying the theory for 2-D structures.

\section{References}

[1] G.T. Tayyar and E. Bayraktarkatal, "Kinematic Displacement Theory of Planar Structures". International Journal of Ocean System Engineering. 2(2) (2012), 63-70.

[2] B.A. Barsky and T.D. DeRose, "Geometric Continuity of Parametric Curves: Three Equivalent Characterizations". IEEE Computer Graphics \& Applications. 9(6) (1989), 60-68.

[3] G.E. Hay, "The Finite Displacement of Thin Rods". Trans. Am. Math. Soc. 51 (1942), 65102.

[4] M. Berger and B. Gostiaux, Differential Geometry: Manifolds, Curves, and Surfaces. New York: Springer, (1988).

[5] J.M. Gere and S.P. Timoshenko, Mechanics of Materials, fourth ed. Boston: Pws, (1997). 